\title{
Effect of whole and clarified egg yolk based extenders on post-thaw semen quality in bulls
}

\author{
Prosper Kamusasa, Eddington Gororo, Fungayi Primrose Chatiza \\ Department of Animal Production and Technology, Chinhoyi University of Technology, Private Bag 7724, \\ Chinhoyi, Zimbabwe
}

\section{Introduction}

Cryopreservation allows long term preservation of gametes for the purpose of conservation or artificial reproduction. However, cooling semen below physiological body temperature causes injury to the sperm cell through mechanical damage due to ice crystallisation and osmotic effects of concentrated solutes in the residual unfrozen water between the ice crystals (Guthrie et al 2002). Sperm cells are generally protected from such cryo-damage using semen diluents with cryoprotective properties. The most commonly used semen extenders for bulls usually contain buffer systems in the form of Tris(hydroxymethyl)aminomethane (Tris) and/or citric acid; metabolizable substrates in the form of fructose or glucose; cryoprotectant molecules, mostly glycerol, egg yolk, milk or soy-lecithin; and antibiotics such as Streptomycin, Penicillin, Polymixin B, Gentamycin, Tylosin, Lincomysin and Spectinomycin (Rauch, 2013; Layek et al 2016).

Chicken egg yolk, included at $20 \%$ in semen extenders, is the non-permeating cryoprotectant of choice in bovine sperm cryopreservation most probably due to its effectiveness, lower cost and ready availability. Egg yolk preserves the integrity of the sperm plasma and acrosomal membranes against temperature-related damage during cooling and freezing owing to the presence of low density lipoproteins (LDL), in association with other components (Purdy, 2006). LDL make up two thirds of the solid fraction of egg yolk. They are spherical molecules of 17-60nm diameter, with triglycerides and cholesterol esters at the core, surrounded by phospholipids and a protein film (Moussa et al 2002). Thus LDLs are composed of approximately $83-89 \%$ lipids and $11-17 \%$ proteins. The LDL's primary protective mechanism is adherence to and stabilisation of the sperm plasma membrane (Andrabi, 2009; Rehman et al., 2013). Another mechanism through which egg yolk protects against cooling injury is that the phospholipids in LDL form a protective film on the sperm surface or replace sperm membrane phospholipids that are lost or damaged during the cryopreservation process (Holt, 2000). Thus, it is the phospholipid moiety of the LDL fraction that has cryoprotective effects in egg yolk.

However, use of egg yolk is associated with a number of disadvantages. Being an animal source ingredient, egg yolk presents significant risk of microbial contamination of the semen, thus necessitating inclusion of antibiotics in egg yolk based semen extenders. Egg yolk is known to 
contain some granular components which are antagonistic to the maintenance of spermatozoal quality and interfere with a number of other biochemical processes (Layek et al 2016). In addition, egg yolk is an extremely complex substance whose composition is variable between batches and changes with diet, and these factors make it impractical to have consistent quality and composition in egg yolk (Moreno et al., 2013). Thus, whole egg yolk shows some toxicity to sperm and interferes with laboratory assays. For these reasons, two major plant derived alternatives to whole egg yolk that have shown potential include soy lecithin or crude soymilk (Singh et al., 2012). In addition, it has been demonstrated by several researchers that egg yolk toxicity can be minimised by reducing concentration of granular and high density material in the media through clarification - repeated centrifugation at low temperature (Amirat et al 2005). Clarification has an advantage of producing a clearer extender that facilitates sperm evaluation and conduct of several sperm biochemical tests (Rauch, 2013).

\section{Objectives}

The experiment was carried out to determine the effects of egg yolk clarification in bovine sperm cryopreservation. Specifically, the study sought (i) to evaluate the comparative cryoprotective effects of whole egg yolk and clarified egg yolk on post thaw sperm quality parameters in Mashona bull semen; and (ii) to determine the optimum clarified egg yolk inclusion level (10-20\%) in semen extenders for Mashona bull semen cryopreservation.

\section{Materials and methods}

Whole egg yolk was prepared by manually breaking fresh hen eggs (Irvine's, Zimbabwe) and separating the yolk from the albumen. Yolks were carefully rolled on a filter paper to remove chalazas and traces of albumen adhering to the vitelline membrane. The vitelline membrane was then disrupted with a needle, whole egg yolk collected into a clean beaker and filtered. To prepare the clarified egg yolk, whole egg yolk was diluted 1:3 (v/v) in double distilled water and centrifuged in sterile tubes at $10,000 \times \mathrm{g}$ for $30 \mathrm{~min}$ at $5^{\circ} \mathrm{C}$. After centrifugation, the pellet was discarded and the water-soluble clear fraction (supernatant) was saved to prepare the clarified egg yolk media. A basic Tris-citric acid extender was modified by the addition of either clarified egg yolk (CEY) or whole egg yolk (WEY) to prepare four experimental extenders as follows: 10\% CEY, 15\% CEY, $20 \%$ CEY and $20 \%$ WEY $(v / v)$.

Two Mashona bulls at the Chinhoyi University Farm were used for the experiment. The research farm is located outside Chinhoyi town, about $120 \mathrm{~km}$ northwest of Harare, Zimbabwe, between $17^{\circ} 21^{\prime} 10.8^{\prime \prime} \mathrm{S}$ latitude and $30^{\circ} 12^{\prime} 21.6^{\prime \prime} \mathrm{E}$ longitude. The farm is located in a warm $\left(20-30^{\circ} \mathrm{C}\right)$, high 
rainfall (750 to 1,000 mm/year, unimodal) sub-tropical area of Zimbabwe (Vincent et al., 1960). The bulls were kept under standard conditions of feeding and management. Semen collection was

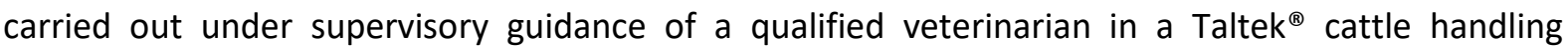
system, and according to the University Guidelines for Animal Handling and Sample Collection. Semen was collected weekly for six weeks from each bull using an electro-ejaculator machine standardised for bovine semen collection (Minitube, Germany). A pre-warmed $\left(35-37^{\circ} \mathrm{C}\right)$ sterile assortment tube connected to a latex rubber cone was used to collect the ejaculate. Soon after, semen was visually assessed for colour, consistency, odour and presence of foreign material.

After collection, each ejaculate was split into four fractions and diluted 1:1 v/v with the four extenders (10\% CEY, 15\% CEY, $20 \%$ CEY and 20\% WEY). The semen was cooled to $4^{\circ} \mathrm{C}$ over two hours. At the laboratory the following parameters were evaluated within 30 minutes of semen collection: concentration, viability, gross motility, progressive linear motility, morphologically normal sperm and occurrence of various sperm defects. Semen was further diluted to a spermatozoa concentration of $100 \times 10^{6}$ per ml at $4^{\circ} \mathrm{C}$, equilibrated for a further 2 hours, packaged in $0.25 \mathrm{ml}$ polyvinyl straws and frozen in liquid nitrogen. After 72 hours straws were thawed for 30 seconds at $37^{\circ} \mathrm{C}$ and evaluated for the same parameters as for fresh semen. Analysis of variance (ANOVA) was carried out in Minitab 17 to compare the effects of cryopreservation, and effects of extender treatment on post-thaw sperm quality variables. Means were separated using Fisher's LSD post hoc tool and declared significant at $\mathrm{P}<0.05$.

\section{Results}

Significant decrease in sperm quality variables analysed were observed following cryopreservation. Semen quality increased with concentration of clarified egg yolk with the three concentrations differing from each other $(P<0.05)$ in post-thaw sperm quality for each parameter tested. This indicated a positive relationship between egg yolk LDL concentration and maintenance of in-vitro sperm quality. The $20 \%$ clarified egg yolk (CEY20) extender treatment gave post-thaw motility, viability and normal morphology values which were comparable to the control (20\% whole egg yolk, WEY20). The $10 \%$ clarified egg yolk concentration gave the least post-thaw quality values and the greatest proportion of defective spermatozoa, showing that it gave the least cryoprotection to the spermatozoa. Contrary to findings in the present study, Amirat et al (2005) observed an advantage of using clarified compared to whole egg yolk in post thaw bull semen quality. A similar study by Rauch (2013) found mixed results. The differences between these studies could be ascribed to methodological differences in the clarification of the egg yolk and the subjectivity of the semen analyses methods used in the present study. 
Table 1 Least Square Means $( \pm S E)$ for various fresh and post thaw semen quality parameters in Mashona bull semen

\begin{tabular}{lcccc}
\hline Variable & Fresh & Post-thaw & P-value & Significance \\
\hline Gross motility & $87.8 \pm 11.94$ & $65.7 \pm 16.65$ & 0.000 & $* * *$ \\
Progressive motility & $78.9 \pm 12.49$ & $57.1 \pm 16.36$ & 0.000 & $* * *$ \\
Viable sperm & $73.2 \pm 17.42$ & $63.9 \pm 18.04$ & 0.052 & $\mathrm{~ns}$ \\
Normal sperm & $74.7 \pm 15.59$ & $58.4 \pm 20.07$ & 0.002 & $* *$ \\
Double tail & $6.0 \pm 6.97$ & $6.2 \pm 6.35$ & 0.916 & $\mathrm{~ns}$ \\
Coiled tail & $4.1 \pm 2.16$ & $6.8 \pm 6.30$ & 0.076 & $\mathrm{~ns}$ \\
Double head & $3.5 \pm 2.84$ & $4.5 \pm 4.35$ & 0.371 & $\mathrm{~ns}$ \\
Microcephalic & $3.3 \pm 2.49$ & $3.7 \pm 3.52$ & 0.672 & $\mathrm{~ns}$ \\
Pyriform (PH?) & $5.9 \pm 6.09$ & $7.4 \pm 8.45$ & 0.481 & $\mathrm{~ns}$ \\
Detached heads & $2.1 \pm 2.01$ & $5.0 \pm 4.27$ & 0.007 & $* *$ \\
\hline
\end{tabular}

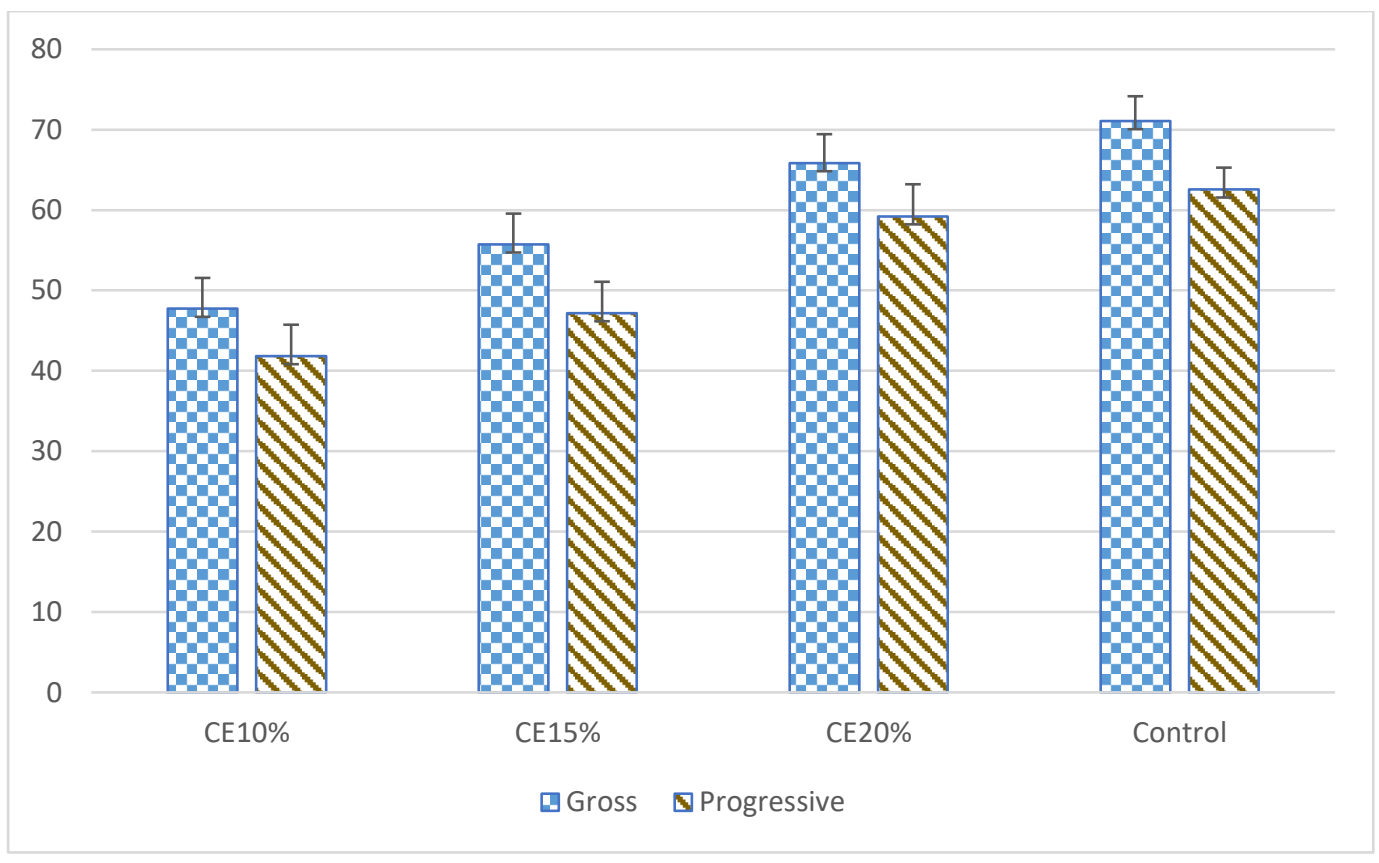

Figure 1: Sperm motility variables of Mashona bull semen frozen in extenders with different concentrations of clarified egg yolk and $20 \%$ whole egg yolk (control) 


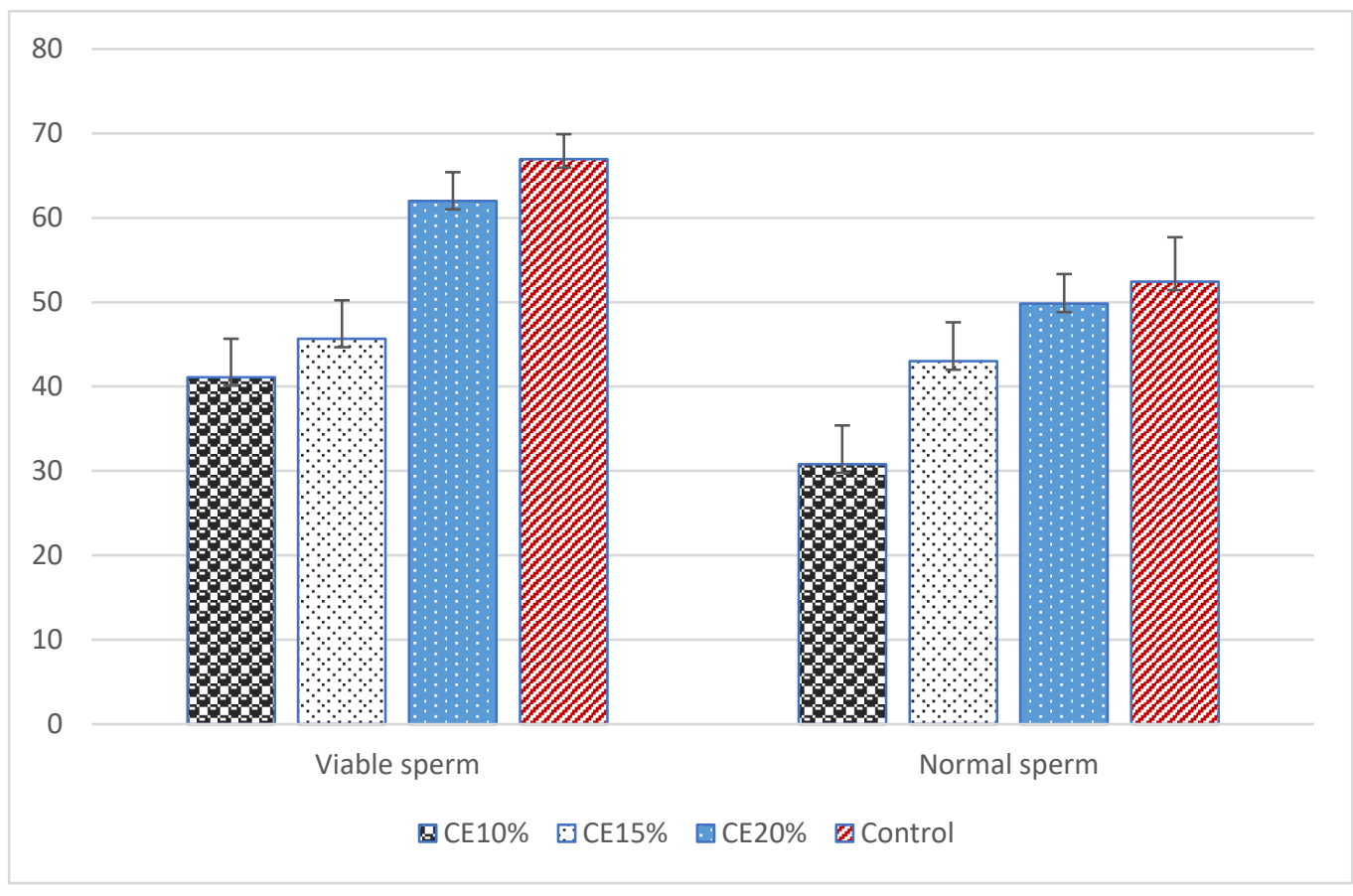

Figure 2: Proportion of viable and morphologically normal sperm in post-thaw Mashona bull semen frozen in extenders with different concentrations of clarified egg yolk and $20 \%$ whole egg yolk (control).

Table 2: Frequency of morphological abnormalities (Mean \pm SE \%) in Mashona bull semen frozen with different concentrations of clarified egg yolk (CEY) and whole egg yolk (WEY)

\begin{tabular}{|c|c|c|c|c|c|}
\hline \multirow{2}{*}{ Sperm defect } & \multicolumn{4}{|c|}{ Egg yolk type and concentration } & \multirow{2}{*}{ P-value } \\
\hline & $10 \%$ CEY & $15 \%$ CEY & $20 \%$ CEY & $20 \%$ WEY & \\
\hline Double tail & $9.8 \pm 1.85$ & $6.2 \pm 0.95$ & $6.4 \pm 1.18$ & $4.8 \pm 1.64$ & $0.093^{\mathrm{ns}}$ \\
\hline Coiled tail & $10.3 \pm 1.79^{\mathrm{a}}$ & $5.0 \pm 0.75^{b}$ & $4.4 \pm 1.18^{b}$ & $4.8 \pm 1.67^{b}$ & $0.012 *$ \\
\hline Broken tail & $8.8 \pm 2.55^{a}$ & $7.8 \pm 1.84^{\mathrm{a}}$ & $3.2 \pm 2.05^{c}$ & $4.9 \pm 1.46^{b}$ & $0.042 *$ \\
\hline Double head & $4.4 \pm 0.88$ & $5.7 \pm 1.48$ & $3.9 \pm 0.96$ & $3.9 \pm 0.64$ & $0.604^{\mathrm{ns}}$ \\
\hline Microcephalic & $5.7 \pm 1.09^{a}$ & $3.2 \pm 0.59^{b}$ & $3.9 \pm 0.73^{\mathrm{ab}}$ & $2.0 \pm 0.63^{b}$ & $0.014 *$ \\
\hline Pyriform (PH?) & $9.5 \pm 2.55$ & $6.9 \pm 1.84$ & $6.8 \pm 2.05$ & $6.4 \pm 1.46$ & $0.686^{\mathrm{ns}}$ \\
\hline Detached heads & $7.1 \pm 1.09^{a}$ & $6.4 \pm 0.71^{\mathrm{a}}$ & $3.2 \pm 0.89^{b}$ & $3.2 \pm 1.01^{b}$ & $0.004^{* *}$ \\
\hline
\end{tabular}




\section{Conclusion}

This experiment found no advantage of replacing whole egg yolk with up to $15 \%$ clarified egg yolk in Mashona bull semen cryopreservation. However, $20 \%$ clarified and $20 \%$ whole egg yolk performed similarly in the maintenance of post-thaw sperm motility, viability and normal morphology.

\section{References}

Amirat, L., Anton, M., Tainturier, D., Chatagnon, G., Battut, I., \& Courtens, J. L. (2005). Modifications of bull spermatozoa induced by three extenders: Biociphos, low density lipoprotein and Triladyl, before, during and after freezing and thawing. Reproduction, 129(4), 535-543

Andrabi, S. (2009). Factors affecting the quality of cryopreserved buffalo (Bubalus bubalis) bull spermatozoa. Reproduction in domestic animals, 44(3), 552-569

Guthrie, H., Liu, J., \& Critser, J. (2002). Osmotic tolerance limits and effects of cryoprotectants on motility of bovine spermatozoa. Biology of reproduction, 67(6), 1811-1816.

Holt, W. (2000). Fundamental aspects of sperm cryobiology: the importance of species and individual differences. Theriogenology, 53(1), 47-58

Layek, S. S., Mohanty, T. K., Kumaresan, A., \& Parks, J. E., (2016). Cryopreservation of bull semen: evolution from egg yolk based to soybean based extenders. Animal reproduction science, 172, 1-9.

Moreno, D., Bencharif, D., Amirat-Briand, L., Neira, A., Destrumelle, S., \& Tainturier, D. (2013). Preliminary results: the advantages of low-density lipoproteins for the cryopreservation of equine semen. Journal of equine veterinary science, 33(12), 1068-1075.

Moussa M, Martinet V, Trimeche A, Tainturier D, \& Anton M., (2002). Low density lipoproteins extracted from hen egg yolk by an easy method: cryoprotective effect on frozen-thawed bull semen. Theriogenology 57, 1695-1706

Purdy, P. (2006). A review on goat sperm cryopreservation. Small Ruminant Research, 63(3), 215225.

Rauch, A. (2013). Cryopreservation of bovine semen in egg yolk based extenders. MSc Thesis, University of Saskatchewan, Canada

Rehman, F. U., Zhaoa, C., Shaha, M. A., Qureshib, M. S., \& Wanga, X. (2013). Semen extenders and artificial insemination in ruminants. Veterinaria, 1(1), 1-8. 
Souza, A. L., Lima, G. L., Peixoto, G. C., Silva, A. M., Oliveira, M. F., \& Silva, A. R. (2016). Use of Aloe vera-based extender for chilling and freezing collared peccary (Pecari tajacu) semen. Theriogenology, 85(8), 1432-1438.*89+++ 